\title{
Supplementary Materials: Reversible Nanoparticle Cubic Lattices in Blue Phase Liquid Crystals
}

\author{
Mohamed Amine Gharbi ${ }^{1,2}$, Sabine Manet ${ }^{1,2}$, Julien Lhermitte ${ }^{2}$, Sarah Brown ${ }^{1,2}$, Jonathan \\ Milette $^{1}$, Violeta Toader ${ }^{1}$, Mark Sutton ${ }^{2}$ and Linda Reven ${ }^{1}$ \\ ${ }^{1}$ Centre for Self-Assembled Chemical Structures (CSACS/CRMAA), Department of Chemistry, McGill University, \\ Montreal, QC H3AOB8. ${ }^{2}$ Department of Physics, McGill University, Montreal, QC H3A2T8.
}

\section{Polarized optical microscopy (POM) characterization}

The different mesophases of the BP mixtures with and without AuNPs were characterized using an upright microscope in transmission mode equipped with crossed polarizers (Motic BA310 Pol), digital color camera (Sony DFW-V500) and temperature controller (LakeShore 336 Temperature Controller with temperature regulated at $0.001{ }^{\circ} \mathrm{C}$ ). A small amount of each BP/AuNP mixture was deposited between two glass slides without spacers and sealed with epoxy glue. The films of BP have a typical thickness around $5 \mu \mathrm{m}$. The functionalized AuNPs fully disperse in the isotropic phase up to particle concentrations of $25 \mathrm{wt}$. \% (Figure S1).
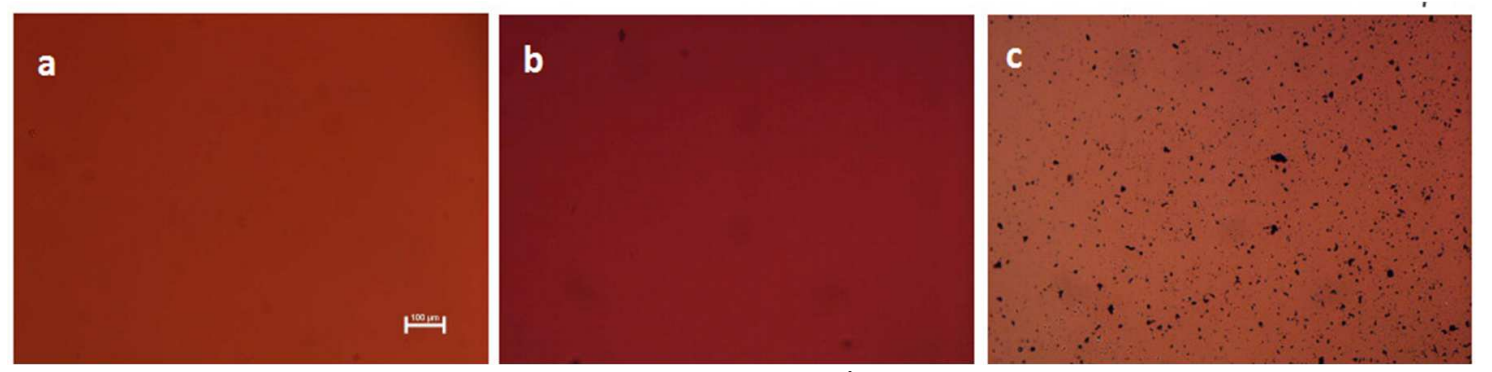

Figure S1: Dispersion of AuNPs in 50:50 mixture of 6OCB/CB15 BP: a- 2 wt.\% of AuNPs, b- 25 wt.\% of AuNPs, and c- 50 wt.\% of AuNPs. The images show that particles are fully dispersed in BP mixtures even at higher concentrations. (Scale bar $100 \mu \mathrm{m}$ ).

Figure S2a shows how well the AuNPs disperse in the isotropic phase of the BP mixture. However, after the isotropic-BP II phase transition, the particles migrate to dislocations that separate the BP platelets as shown in Figure $\mathbf{S 2} \mathbf{b}$. Over time, these platelets grow and coalesce as the temperature decreases and AuNPs diffuse within the 3D network of BP defects (i.e. they redisperse into the 3D cubic network of the BP defects).
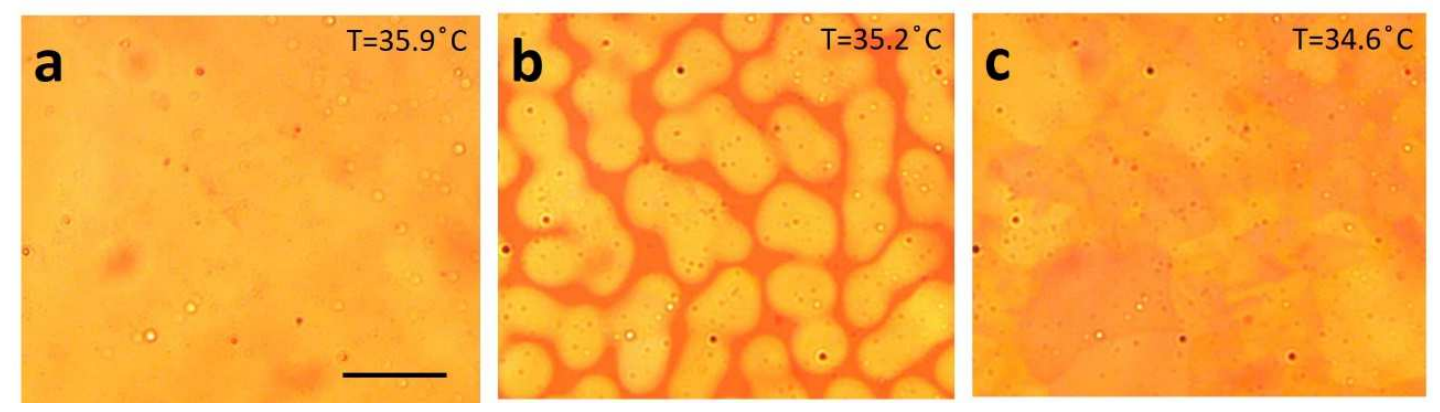

Figure S2: Dispersion of AuNPs in BP disclinations. The BP mixture is 55:44 6OCB/CB15 and the particle concentration is $2.5 \mathrm{wt} . \%$. a- In the isotropic phase, particles are fully dispersed. b- After the isotropic-BP 
II phase transition, NPs aggregate between the BP platelets then, c- slowly diffuse in BP disclinations.

(Scale bar $100 \mu \mathrm{m}$ ).

Finally, once the cholesteric phase forms, the AuNPs are expelled from the LC phase and form large aggregates as shown in Figure $\mathbf{S 3 .}$

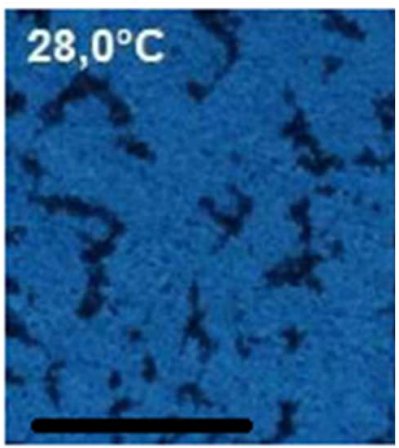

Figure S3: Formation of aggregates of AuNPs at $\mathrm{T}=28^{\circ} \mathrm{C}$ in the cholesteric phase. (Scale bar $50 \mu \mathrm{m}$ ).

Using POM characterization, the phase diagrams of the mixtures as a function of the ratios of $60 C B$ and $\mathrm{CB} 15$ as well as the AuNP concentration were explored. The phase transition temperatures decrease and BP stability temperature range widths increase as a function of AuNP concentration. Table S1 summarizes the effect of AuNP concentration on the 50:50 60CB/CB15 mixture.

\begin{tabular}{|c|c|c|c|c|c|}
\hline AuNPs wt.\% & AuNPs vol.\% & Ramp $\left({ }^{\circ} \mathrm{C} / \mathrm{min}\right)$ & Iso-BP II $\left({ }^{\circ} \mathrm{C}\right)$ & BP II-BP I $\left({ }^{\circ} \mathrm{C}\right)$ & BP I-Chol $\left({ }^{\circ} \mathrm{C}\right)$ \\
\hline 0 & 0 & 0.2 & 31 & 30.4 & 29.7 \\
\hline 0.9 & 0.05 & 0.2 & 31.2 & 30.9 & 29.3 \\
\hline 10 & 0.5 & 0.2 & 29.8 & 28.9 & $<24.5$ \\
\hline 20 & 1.03 & 0.2 & 28.9 & 27.9 & 23 \\
\hline
\end{tabular}

Table S1: POM study of the BP formation for 50:50 6OCB/CB15 mixture as a function of AuNP concentration.

\section{Reflectance measurements}

Blue phases are characterized by their self-assembled periodic defect structures that exhibit 3D cubic symmetry. Like photonic crystals, the cubic periodic structures of blue phases results in optical Bragg diffraction. For a cubic crystal with a lattice constant $a$, Miller indices $h, k$, and $l$, the reflection wavelength can be expressed as:

$$
\lambda=\frac{2 n a \cos \alpha}{\sqrt{h^{2}+k^{2}+l^{2}}}
$$

Here $n$ is the average refractive index of the liquid crystal and $\alpha$ denotes the incident angle of light with respect to the crystal plane. 
To explore the effect of AuNP concentration on the BP lattice constants, the Bragg reflections in BP I and BP II were measured. A Spectroscopic Variable Angle Ellipsometer (Sopra VASE) was adapted for reflectance measurements (Figure S4).
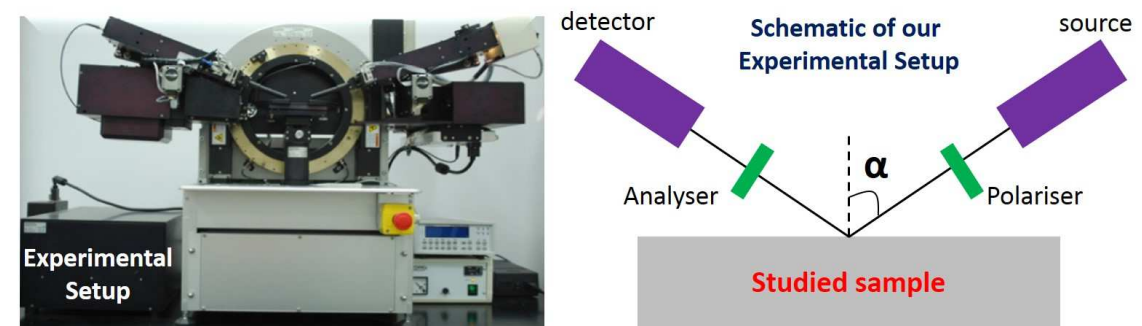

Figure S4: Spectroscopic Variable Angle Ellipsometer adapted for reflectance measurements (left) and its corresponding schematic (right). The incident angle $\alpha$ used in all measurements is equal to $\alpha=45^{\circ}$.

The experimental setup is equipped with a temperature controller (LakeShore 336 Temperature Controller with temperature regulated at $0.001^{\circ} \mathrm{C}$ ) for measuring the temperature dependant reflection spectra of our blue phase samples prepared with different AuNP concentrations. It involves illuminating samples with a collimated microbeam of polarized light then analyzing the reflected beam with the same polarization, to measure the reflectance of light as a function of wavelength. Figure S5 shows the measured reflectance with and without AuNPs. It confirms the fact that AuNPs arrange themselves in the defect network of the blue phase without changing the shape or parameters of the cubic structure. We note that all experiments are realized at an incident angle $\alpha=45^{\circ}$. By measuring the wavelengths of the Bragg peaks, the lattice constants of the blue phase were estimated and their values are in good agreement with lattice constants measured by SAXS (around $360 \mathrm{~nm}$ ).
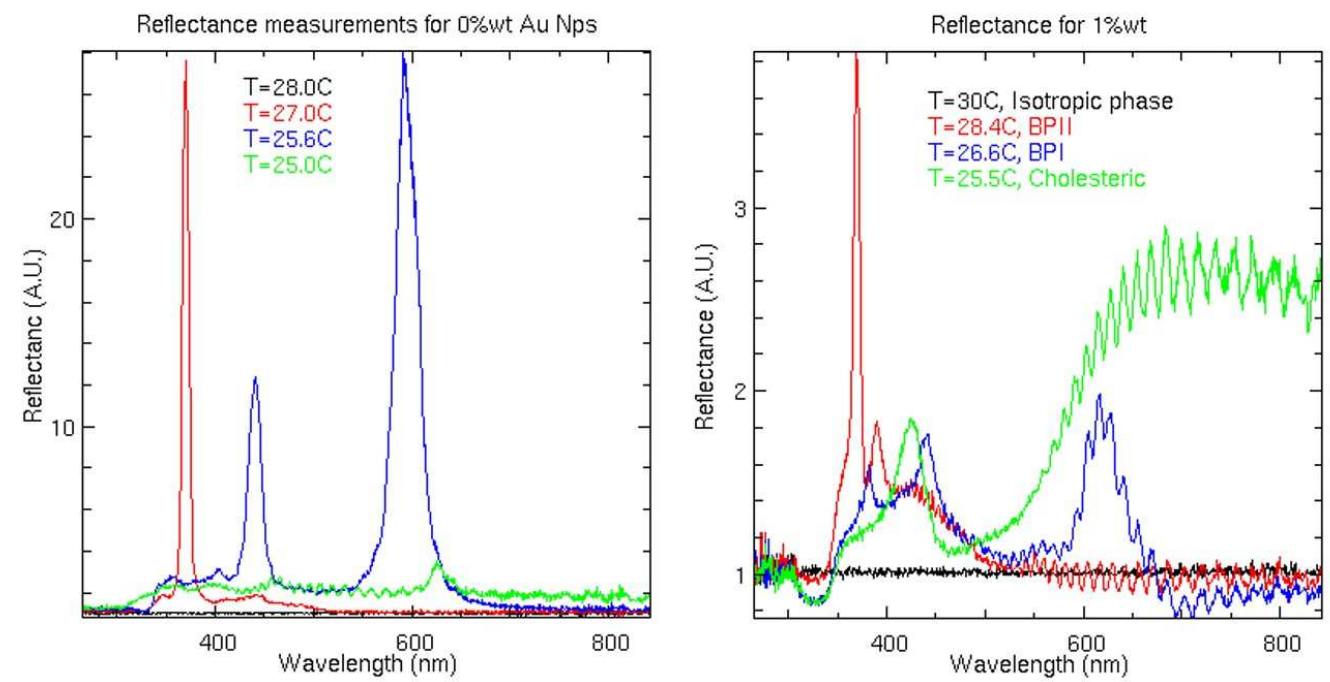

Figure S5: Reflectance measurements of the Bragg peaks of 50:50 60CB/CB15 mixture. Left: without AuNPs. Right: with $1 \mathrm{wt. \%}$ AuNPs. The Bragg peaks are at approximately the same positions. The black indicates the isotropic phase, the red is the BP II, the blue is the BP I and the green color is the cholesteric phase.

\section{Small angle x-ray scattering (SAXS) measurements}

The X-ray measurements were conducted at the beamline at Sector 8-ID-I of the Advanced Photon Source (APS) in Argonne National Laboratories (USA). The samples were filled in capillary tubes and 
placed in a sample chamber under vacuum $10^{-4}$ torr for thermal isolation. They are then illuminated by a $20 \mu \mathrm{m} \times 20 \mu \mathrm{m}$ square X-ray beam of uniform intensity in transmission geometry. The incoming flux was $\sim 3.7 \times 10^{9}$ photons/s. The X-ray wavelength was $1.62 \AA$ and the $q$ range probed spanned from 0.002 to $0.017 \AA^{-1}$. The X-ray data was collected after heating BP mixtures above the isotropic-BP II temperature transition to erase any thermal history and disperse the AuNPs. Samples are then cooled slowly. After allowing the sample to equilibrate for at least 10 minutes, several measurements were taken for each temperature at different spots along the capillary to avoid radiation damage of the sample. In some cases, data was collected while cooling the system at $1^{\circ} \mathrm{C}$ per minute from the isotropic phase to the cholesteric phase to explore the assembly of AuNPs with the liquid crystal mesophases.

SAXS was used to evaluate the structure of the AuNPs in BP mixtures. We note that in our experiments, only the scattering from AuNPs is detected and not the BP. The Bragg peaks are only observed in presence of AuNPs (no peaks observed in the pure BP mixture as shown in Figure S6). As described in section 3, the spacing $d_{h, k, l}$ between adjacent lattice plane denoted by Miller indices $h, k$, and $l$ is given by eq. 1. For cubic systems, the expression of the lattice constant is given by:

$$
a=\frac{2 \pi}{q} \sqrt{h^{2}+k^{2}+l^{2}},(\text { eq. } 2)
$$

where $q$ is the average wave vector of the Bragg peaks. Thus, the measure of $q$ as a function of temperature enabled us to deduce the variation of the lattice constants of each BP mesophase based on eq. 2. Figure $\mathbf{S 7}$ shows an example of fitting the $q$ positions of Bragg peaks. Table S2 shows an example of measuring $a$ as a function of temperature. We note that using SAXS we can also precisely determine the phase diagram of our BP mixtures. Table S3 represents the AuNP concentration dependence of the lattice constant.

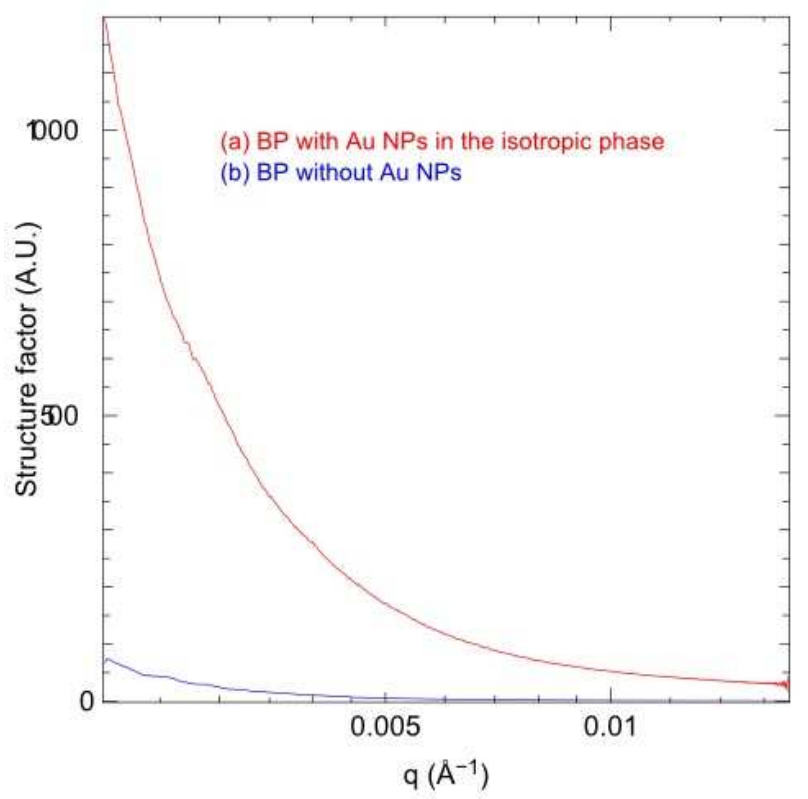

Figure S6: The isotropically averaged scattered intensities in the BP (50:50 6OCB/CB15 mixture) with 1 wt.\% AuNPs (red) at $\mathrm{T}=35^{\circ} \mathrm{C}$ (isotropic phase) and BP without AuNPs (blue) at $\mathrm{T}=30^{\circ} \mathrm{C}$ (BP II). The data show that in absence of AuNPs, the Bragg peaks are not observed. 


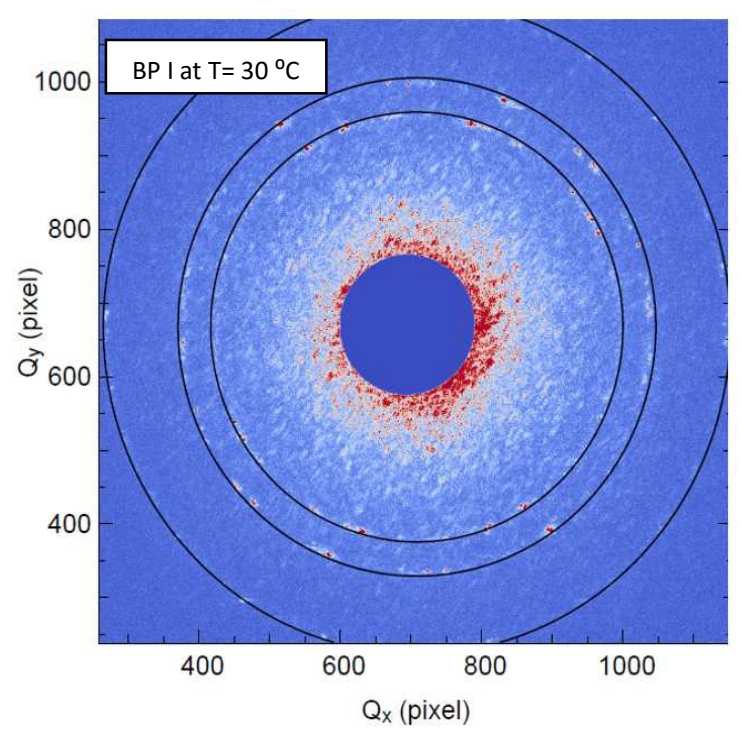

Figure S7: Measurement of $q$ by fitting Bragg peaks with concentric circles for 50:50 60CB:CB15 mixture with 1 wt.\% AuNPS (BP I at T=30 ${ }^{\circ} \mathrm{C}$ ). The best value of $h, k$ and $/$ was chosen to give approximately the same lattice constant $a$ using eq. 2.

\begin{tabular}{|c|c|c|}
\hline Temperature $(\circ \mathrm{C})$ & Lattice constant $(\mathrm{nm})$ & BP mesophase \\
\hline 31.3 & 354.8 & BP II \\
\hline 31.1 & 352.1 & BP II \\
\hline 30.9 & 350.8 & BP II \\
\hline 30.8 & 351.3 & BP I+BP II \\
\hline 30.7 & $252.8 / 347.3$ & BP I+BP II \\
\hline 30.5 & $352.5 / 347.7$ & BP I+BP II \\
\hline 30.3 & $355.6 / 348.7$ & BP I \\
\hline 30.0 & 350.7 & BP I \\
\hline 29.8 & 355.5 & BP I \\
\hline 29.6 & 353.5 & BP I \\
\hline 29.2 & 365.3 & BP I \\
\hline 28.5 & 365.2 & BP I \\
\hline 28.0 & 363.7 & BP I \\
\hline 26.0 & 363.3 & \\
\hline & & \\
\hline
\end{tabular}


Table S2: Lattice constant of the cubic structures formed by 0.9 wt $\%$ AuNPs in 50:50 mixture of 60CB/CB15 measured using SAXS.

\begin{tabular}{|c|c|}
\hline AuNP concentration & Average lattice constant (nm) \\
\hline 1 wt.\% & 355.3 \\
\hline $10 \mathrm{wt.} \%$ & 378.4 \\
\hline
\end{tabular}

Table S3: AuNP concentration dependence of BP lattice constant for 50:50 mixture of 60CB/CB15.

\section{Calculation of the structure factor: Powder Peaks}

From Warren (eq. 4.12, B.E. Warren, X-ray Diffraction, Dover (1990)) the integrated intensity for Bragg peaks from a powder is proportional to:

$$
I_{h k l} \propto \frac{m_{h k l}\left|F_{h k l}\right|^{2}}{\sin ^{2}\left(\theta_{h k l}\right) \cos \left(\theta_{h k l}\right)},(e q .3)
$$

where $m_{h k l}$ is the multiplicity of the Bragg peak hkl, and $\left|F_{h k l}\right|^{2}$ is the structure factor of the unit cell at the wavevector $q$ having scattering angle $2 \theta_{h k l}$ corresponding to the Bragg peak. For a spherical $\mathrm{Au}$ nanoparticle the atomic structure factor is proportional to:

$$
\left.F_{\text {sphere }}(q)=3 \frac{\sin (q R)-q R \cos (q R)}{(q R)^{3}}, \text { (eq. } 4\right)
$$

where $R$ is the sphere radius. From the existence of the $(2,2,0)=(1,1,0)(\mathrm{sc})$ peak (multiplicity 12$)$ we infer that either the Wycoff sites $(0,0,0)$ or $(x, x, x)$ are occupied. These give a contribution to the structure factors of twelve times 4 or twelve times $16(1+\cos (4 \pi x))^{2}$. The existence of the $(2,2,2)=(1,1,1)$ (sc) peaks (multiplicity 8 ) means the Wycoff sites $(1 / 4,1 / 4,1 / 4)$ are populated and these have contribution of eight times 16. Each of these factors would be multiplied by the fractional occupancy of the sites. The larger intensity of the $(220)$ peaks suggests that the $(1 / 4,1 / 4,1 / 4)$ sites have much smaller occupancies.

For the BPI structure we were unable to come up with a simple model to predict the positions of the gold particles. The overall increased intensity of the scattering in the BPI phase suggests a higher concentration of the AuNPs in the structure. Our attempts to guess the occupancy included chosing Wycoff sites along the disclination lines and testing all combinations of filling these sites with AuNPs. We also considered several occupancy combinations in the BPII phase, extending them to the $2 \times 2 \times 2$ lattice which would fill the BPI unit cell. None of the combinations we tried gave a (221) or (300) peaks as the lowest occuring Bragg peak.

\section{AuNP dynamics}

No change in dynamics from the gold nanoparticles in the blue phase was observed. The dynamics are quantified through the covariance in time of the intensity versus position in the detector $\vec{q}$ and time lag $\tau$ : 


$$
g_{2}(\tau)=\frac{\langle I(\vec{q}, t) I(\vec{q}, t+\tau)\rangle_{t, \Delta \vec{q}}}{\langle I(\vec{q}, t)\rangle_{\Delta \vec{q}}\langle I(\vec{q}, t+\tau)\rangle_{\Delta \vec{q}}},(e q .5)
$$

where the $t$ subscript signifies an average over all time and a $\Delta \vec{q}$ subscript signifies an average over a small area of the detector (for example, a circular average here). The latter is usually performed to help obtain better statistics of the signal.

A brute force calculation of this formula was performed. This allowed one to quantify the fluctuation of the speckle, which is related to the fluctuation of the system. The scattered speckled is measured solely from the gold, which was determined with comparison of samples without gold. The life time of the correlation function quantifies the speed of these fluctuations. They were observed to be too fast to measure with our detector's time resolution in the isotropic and blue phases. The fluctuation of the speckle will also vary with momentum transfer wavevector $q$. Speckle from diffusive motion will decorrelate exponentially in time, with this exponential time constant related to $1 / q^{2}$ [Dierker et al., Physical review letters, 75(3):449, 1995]. Speckle from subdiffusive or superdiffusive motion will decorrelate exponentially in time with a time constant related to $1 / q$ [Cipelletti et al., Faraday Discussions, 123:237-251, 2003]. An intermediate power law $\alpha$ was observed, suggesting that it is not a simple diffusion. An example can be seen in Figure S8. The correlation functions have all decayed with the exception of low $q$. Rings of $q$ away from the Bragg peaks from the blue phase were purposely chosen.

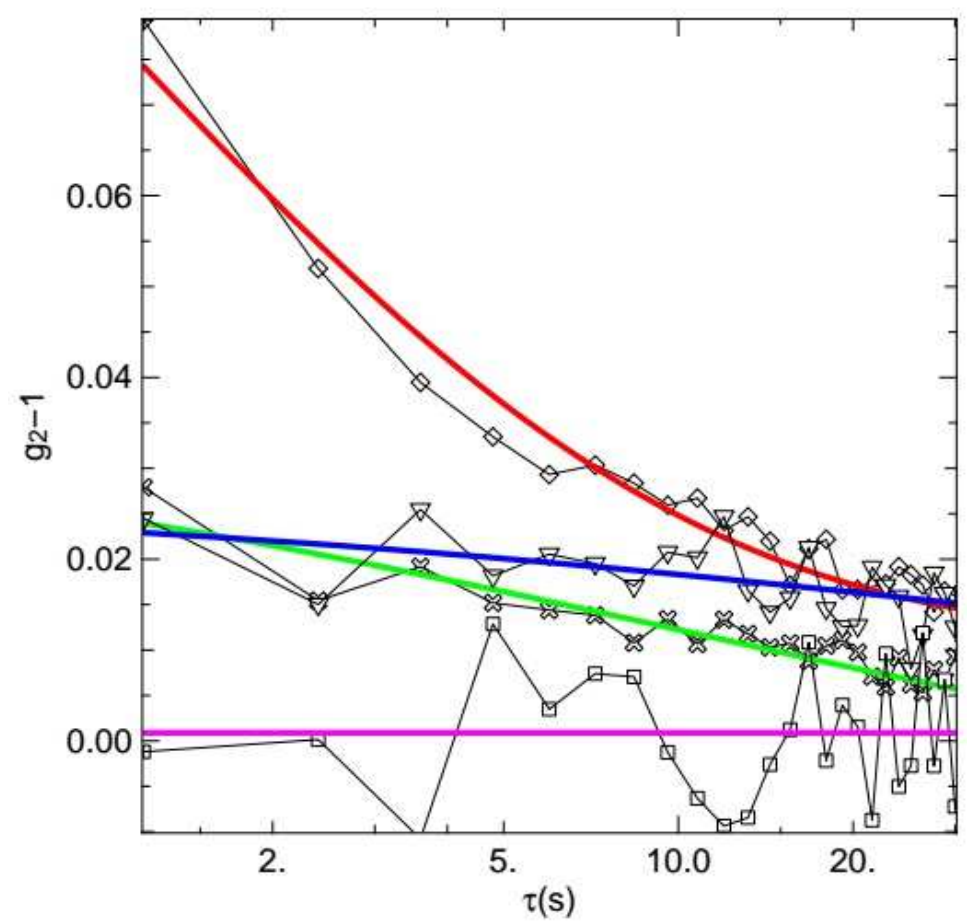

Figure S8: A plot of the dynamics observed for $q\left(\right.$ in $\left.\AA^{-1}\right)=2.33 \times 10^{-3}$ (red), $3.93 \times 10^{-3}$ (green),

$6.06 \times 10^{-3}$ (blue), and $9.25 \times 10^{-3}$ (magenta), at $\mathrm{T}=30.3^{\circ} \mathrm{C}$ (the phase is BP II) of the $50: 5060 \mathrm{CB} / \mathrm{CB} 15$ mixture with 1 wt.\% AuNPs.

The speckle fluctuating at the lowest $q$ will fluctuate the slowest. The decorrelation of the speckle at this $q$ was measured in the isotropic, blue phases and cholesteric phases (figure S9). It was found that the 
dynamics both in the blue phase and isotropic phase were too fast to measure. As the sample approached the cholesteric phase, a correlation function with a time constant within the time resolution of the detector was measured. Due to the speed of the fluctuations in the blue phase and isotropic phase, it is not possible to infer any conclusions on the dynamics of the gold.

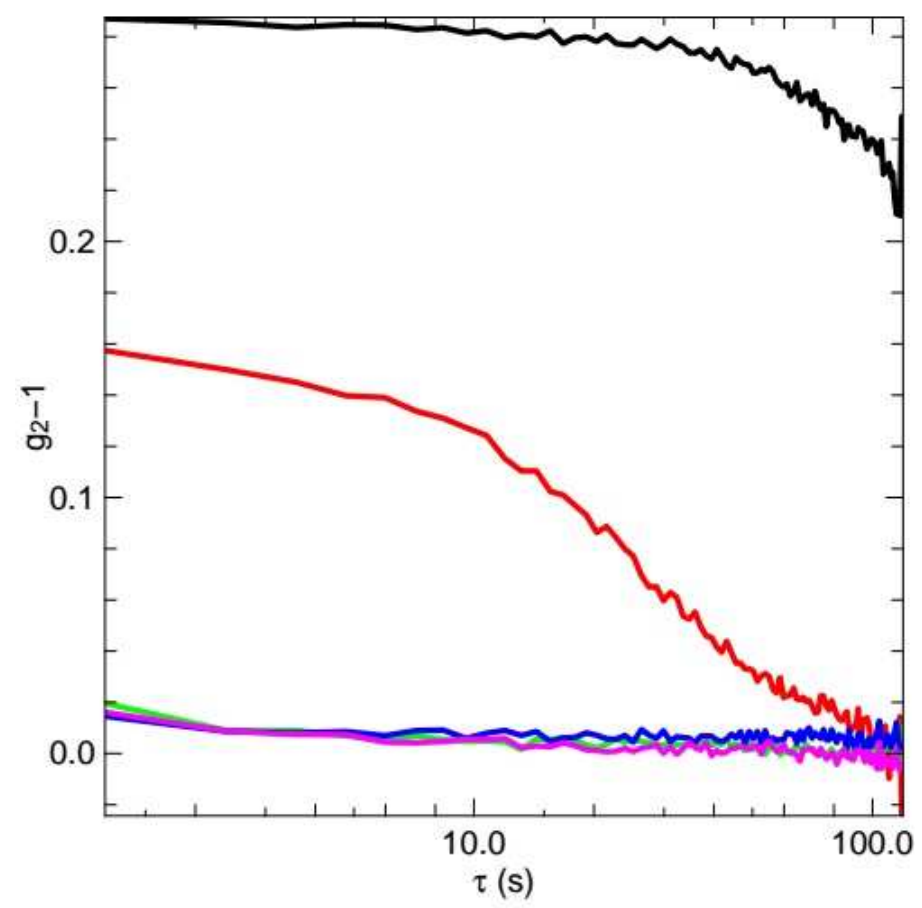

Figure 59: A plot of some of the correlation functions at $2.33 \times 10^{-3} \AA^{-1}$, for $\mathrm{T}=28^{\circ} \mathrm{C}$ (black, the phase is cholesteric), $30.2^{\circ} \mathrm{C}$ (red, the phase is BP I), $30.9^{\circ} \mathrm{C}$ (green, the phase is BP II), $31 .{ }^{\circ} \mathrm{C}$ (blue, the phase is isotropic), $31.8^{\circ} \mathrm{C}$ (magenta, the phase is isotropic) of the 50:50 6OCB/CB15 mixture with 1 wt.\% AuNPs. 